\title{
Integration of new features for telerobotic surgery into the MiroSurge system
}

\author{
Rainer Konietschke ${ }^{\mathrm{a}, *}$, Davide Zerbato ${ }^{\mathrm{b}}$, Rogério Richa ${ }^{\mathrm{c}}$, Andreas Tobergte ${ }^{\mathrm{a}}$, Philippe Poignet ${ }^{\mathrm{c}}$, \\ Florian A. Fröhlich ${ }^{\mathrm{a}}$, Debora Botturi ${ }^{\mathrm{b}}$, Paolo Fiorini ${ }^{\mathrm{b}}$ and Gerd Hirzinger ${ }^{\mathrm{a}}$ \\ ${ }^{a}$ Institute of Robotics and Mechatronics, German Aerospace Center (DLR), Wessling, Germany \\ ${ }^{\mathrm{b}}$ Department of Computer Science, University of Verona, Verona, Italy \\ ${ }^{\mathrm{c}}$ Robotics Department, LIRMM CNRS/Université Montpellier II, France
}

\begin{abstract}
Minimally invasive robotic surgery has gained wide acceptance recently. Computer-aided features to assist the surgeon during these interventions may help to develop safer, faster, and more accurate procedures. Especially physiological motion compensation of the beating heart and online soft tissue modelling are promising features that were developed recently. This paper presents the integration of these new features into the minimally invasive robotic surgery platform MiroSurge. A central aim of this research platform is to enable evaluation and comparison of new functionalities for minimally invasive robotic surgery. The system structure of MiroSurge is presented as well as the interfaces for the new functionalities. Some details about the modules for motion tracking and for soft tissue simulation are given. Results are shown with an experimental setup that includes a heart motion simulator and dedicated silicone organ models. Both features are integrated seamlessly and work reliably in the chosen setup. The MiroSurge platform thus shows the potential to provide valuable results in evaluating new functionalities for minimally invasive robotic surgery.
\end{abstract}

Keywords: Medical robotics, minimally invasive robotic surgery, soft tissue simulation, heart motion tracking and compensation

\section{Introduction}

Robotic assistance in minimally invasive surgery has been a promising approach for many years. But only recently it achieved wide acceptance in e.g. radical prostatectomy due to advantages like less invasiveness and a shorter hospital stay [35]. The only system for these interventions which is commercially available today is the daVinci ${ }^{\circledR}$ product line of Intuitive Surgical ${ }^{\circledR}$. It is structured into a patient-side slave cart and a master console to teleoperate the slave. The robotic slave side offers up to four arms, dex-

*Corresponding author: Rainer Konietschke, Institute of Robotics and Mechatronics, German Aerospace Center (DLR), Münchnerstrasse 20, 82234 Wessling, Germany. E-mail: Rainer. Konietschke@dlr.de. trous instruments and stereo imaging. On the master side, the surgeon gets a stereo vision of the scene and commands the slave side via hand controllers, buttons and foot pedals. The telemanipulation is enhanced by additional features like motion scaling and tremor filtering. Various research platforms were developed up to now that aim at extending these functionalities in different ways, see e.g. $[45,17]$ This paper presents as a result of the European project AccuRobAs the integration of two new features that are widely considered beneficial. Motion compensation controls the robotic system in a way that the surgeon may operate on a virtually still standing region of interest (ROI) on an organ. Online integration of soft tissue simulation makes a virtual preview of certain operation steps possible. Both features did not have strong relevance in 
industrial robotics until now, where usually the workpiece was in rest, or had a well known motion, and could be described by simple and known material properties. However, the relevance of these features in the context of surgical robotics is evident. Recently, features that enable to work in unstructured workcells also gain relevance in industrial robotics e.g. when cooperating with the human. In surgical teleoperation, the benefits that come from the physical simulations are manifold [5] The virtual environment can be used during a pre-operative planning phase but it is also useful to integrate supplemental information during the actual intervention. In particular augmented reality obtained with the superimposition of the simulated scene over the real camera view can help the surgeon in navigating the operating area by highlighting important or hidden structures (e.g. vessels, tumors). With virtual fixtures certain areas can be prohibited. In addition, the forces computed by the physical simulation can be used to warn the user about wrong or dangerous gestures or rendered to the user to increase perception of the real environment [23].

This paper describes the integration of the features motion tracking of the beating heart and online soft tissue modelling into the MiroSurge research platform shown in Fig. 1. The MiroSurge platform, developed at the German Aerospace Center (DLR), enables research on minimally invasive robotic surgery. It follows a modular design both on slave and master side. On slave side (see Fig. 1, left), MIRO robots [16] realize the extracorporal motion for positioning two instruments and a stereo endoscope. Inside the body, the instruments are equipped with a three degree of freedom (DoF) actuation to allow for full dexterity and an additional function such as gripping. The tip of the instrument furthermore integrates a seven DoF sensor to measure contact forces and torques as well as grip- ping forces. The forces are fed back to the master side (see Fig. 1, right) either for visual augmentation or for haptic rendering with a device such as the shown commercially available omega.7 input devices [11].

Various features to assist the surgeon and to increase the operation quality are currently researched. These features either aim to reduce the system's complexity (e.g. setup planning), increase the patient safety (e.g. virtual walls), enhance the surgeon accuracy (e.g. intelligent motion scaling), or provide additional information (e.g. visual augmentation, or force feedback). Research on the MiroSurge platform intends to include all these aspects. Namely, an optimization and planning software assists the surgeon during system setup and patient registration [22], and the surgeon receives force feedback during the operation, either visually augmented or directly rendered to the haptic input devices [16]. The features motion tracking of the beating heart and online soft tissue modelling have the potential to further enhance the MiroSurge system and are introduced in the next paragraphs.

Motion tracking. Although current surgical platforms have considerably improved the ergonomics and mobility issues related to the minimally invasive procedure, physiological motion still needs to be manually compensated by the surgeon. For tackling this issue, an active motion compensation can be employed for enabling surgeons to operate directly on moving organs with high accuracy and low cognitive burden.

Unlike passive motion compensation solutions such as mechanical stabilizers, active motion compensation systems are based on the online estimation of physiological motions. Solutions vary from mechanical systems such as the moving support proposed by Trejos et al. [48] to augmented reality systems [42], where a virtually stable view is rendered for the surgeon. All studies report a significant increase in the precision
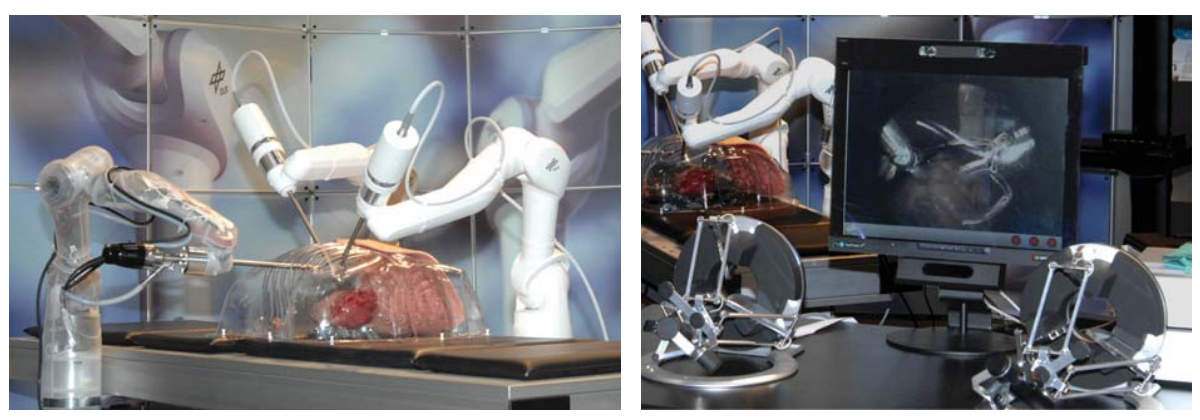

Fig. 1. The MiroSurge System: Slave side (left) and master side (right). 
and repeatability of the surgical gesture, attesting the practical value of a motion compensation system in robotized surgery.

A fundamental requirement in a motion compensation system is the accurate estimation of physiological motions. In the system that integrates the MiroSurge platform, the visual feedback provided by the endoscope is used for motion tracking. From a practical point of view, passive vision-based techniques are preferred for avoiding contact and the introduction of additional sensors in the limited surgical workspace. To the authors' knowledge, this is the first vision-based 3D motion compensation system to incorporate a surgical platform that does not require the insertion of artificial markers for facilitating the visual tracking task.

In the context of motion compensation, cardiac surgery represents the most challenging scenario inside the human body due to the complex heart dynamics and high precision requirements. In literature, several visual tracking approaches for estimating the beating heart motion can be found. Techniques can be divided into two classes: feature-based or region-based tracking.

Feature-based methods [44] can be used to track salient features such as blood vessels or other distinguishable structures for retrieving the heart motion. Although various studies suggest the feasibility of motion estimation using this class of methods, tracking has limited performance against large tissue deformations and appearance changes. Furthermore, it is strongly dependent on the availability of stable features. Its main advantage is the low computational requirements, which enables real-time performance.

On the other hand, in region-based methods such as [36] tracing is formulated as an iterative registration problem. Given the assumption that the heart surface is smooth, continuous and sufficiently textured, a parametric function can be used to describe the heart surface deformation. Region-based methods first appeared as methods for estimating depth in the intraoperative field rather than 3D motion tracking. Lau et al [24] proposed a B-spline parametric model to retrieve depth from the disparity between stereo images of a beating heart. Another approach for dense 3D depth recovery was proposed by Stoyanov et al. [43] where a piecewise bilinear mapping is applied for modeling soft-tissue deformation. A challenge when using these methods is the handling of specular highlights which requires additional preprocessing [14].
The issues raised above concern the complicated problem of finding a suitable deformation model for the heart surface. A possible solution can be physically inspired models such as the membrane model proposed by Bader et al. [2]. However, convincing experimental results are still needed to demonstrate the validity of such models. In addition, results of shapefrom-shading (SfS) techniques for reconstructing the heart surface have also been reported in Lo et al. [30, 31]. Compared to the computation stereo approaches presented earlier which perform well in regions with distinctive features and sufficient texture, shape-fromshading tends to perform well in regions with uniform albedo, little texture and smooth local curvature. Since SfS and computational stereo techniques are complementary, a probabilistic fusion framework based on Markov Random Fields (MRF) was proposed. The integration of human perception through gazecontingence in a similar fusion framework was recently proposed in Visentini-Scarzanella et al. [49]. Nevertheless, the uniform albedo and Lambertian surface requirement for SfS to provide reliable results are not often met in MIS images. Furthermore, the computational requirements are still an issue when real-time performance is envisaged.

From the methods cited above, a region-based method based on the Thin-Plate Spline (TPS) deformable model was chosen for the motion compensation system. The most interesting feature of the TPS model is the flexibility in parameterizing deformations, which allows us to overcome problems concerning the lack of visual information in regions with poor texture. Tracking is formulated as an iterative registration problem using a gradient-based minimization technique called ESM [8] extended to the stereo scenario for tracking in 3D. Further implementation details are given in Section 2.2.

Online soft tissue modelling. The simulation of deformations represents an important aspect in many fields. From structural analysis to 3D modeling the need for realistic or fast simulation of deformations lead to the development of many different approaches. In the last few years, increased computational power and advances in research lead to the introduction of soft or deformable models into virtual interactive environments. In the medical field this translates into more realism for surgeon training or intervention planning. A key aspect of interactive deformable models is the computational complexity, and thus the optimized implementation of the simulation. For this reason many 
different models were developed and some parallel implementations were proposed.

Deformable models can be roughly divided into three main classes: finite element models (FEM), meshless models and mass spring models $(M S M)$. Two main issues arise from simulation of deformable models: physics realism requires small temporal steps in the simulation, in addition haptic feedback should be updated every $1 \mathrm{msec}$ to be smooth and realistic, thus interactive simulations with haptic feedback impose very tight constraints on the computational time and require optimized implementations.

FEMs can give the most accurate results in simulations of deformable tissues, they are based on a continuum representation of the tissue and on material constitutive equations [12]. This makes them realistic and easy to calibrate. Their use is widespread in offline engineering and surgery simulations where accuracy is achieved at high computational cost [20]. Linear FEM are used in online simulation but their realism is reduced to small deformations and rotations [6].

Meshless models (or point based methods) share the theoretical background with FEM and have been used in simulation of different materials [34, 19] and also in surgical simulations [27, 29]. The main advantage of point based models is their ability to handle cuts, and more generally, changes in topology. Along with their high computational cost, one of the main drawbacks of point based models is the difficulty in handling surface and interactions because modelling of physics and surface is decoupled.

A different approach leads to MSMs [37] which have been widely used in surgical simulations $[1,33]$. In $M S M s$ the body is discretized in a cloud of mass points linked by springs and dampers. A displacement in a mass position leads to an extension or a compression of one or several springs. This produces internal forces that are integrated temporally and leads to a different configuration of the body. The main disadvantage using $M S M$ is the difficulty in their calibration, since their physical background is not as evident as FEM and the definition of spring and damper coefficients is not straightforward. Their reduced computational complexity makes them suitable for interactive, physically based, simulation of very complex models, moreover $M S M s$ have been effectively ported on graphics processing units (GPUs), fully exploiting their parallelism and memory management. For these reasons the work presented here focuses on the use of MSM in conjunction with a $G P U$.
Many works have been proposed about the use of the $G P U$ to speed up computations of deformable environments simulation $[18,50]$. An implementation of $M S M$ on $G P U$ is presented in [13]: masses are allocated on a $2 \mathrm{D}$ texture and springs on a group of 2D textures where each element stores a spring connected to a mass stored on the same position. In [1] a method for physical simulation that relies on GPU and on MSMs to mimic in real time the physics of human organs is presented. The work proposes an innovative node ordering method and optimized spring forces computation that allow to obtain online simulation.

The remainder of this paper is organized as follows: In Sec. 2, the developed algorithms are presented, namely the integration into the MiroSurge system (Sec. 2.1.), the motion tracking (Sec. 2.2.), and the online soft tissue modelling (Sec. 2.3.). Preliminary experimental results are shown in Secs. 3, 4 concludes the paper.

\section{Methods}

The next Subsection describes how the new features are embedded into the Mirosurge control scheme. Then, the modules Tracking of the beating heart and Soft Tissue Modelling are further detailled.

\subsection{Integration into MiroSurge}

The features presented in this paper need to be integrated into the basic scheme of the so far implemented force feedback telemanipulation control shown in Fig. 2. The bilateral telemanipulation implements a position-force architecture with motion scaling. Cartesian velocities measured at the master are scaled down by a factor of 2-4 and integrated on the slave's initial pose. The motions from the haptic device are transformed in a way that an intutive hand-eye-coordination is given for the user in the sense that moving of the haptic device in one direction corresponds to the motion the user sees in the stereo image. The measured interaction forces returned from the slave are amplified and commanded to the master. The gain factor depends on the motion scaling [47]. An analytic inverse kinematics for the MIRO and the instrument calculates the joint angles such that the instrument crosses the entry point and reaches the commanded slave pose. The analytic solution is enhanced by a numeric optimization for the 1 DoF nullspace to avoid collision of the robotic arms, singularities and joint limits [21]. The 


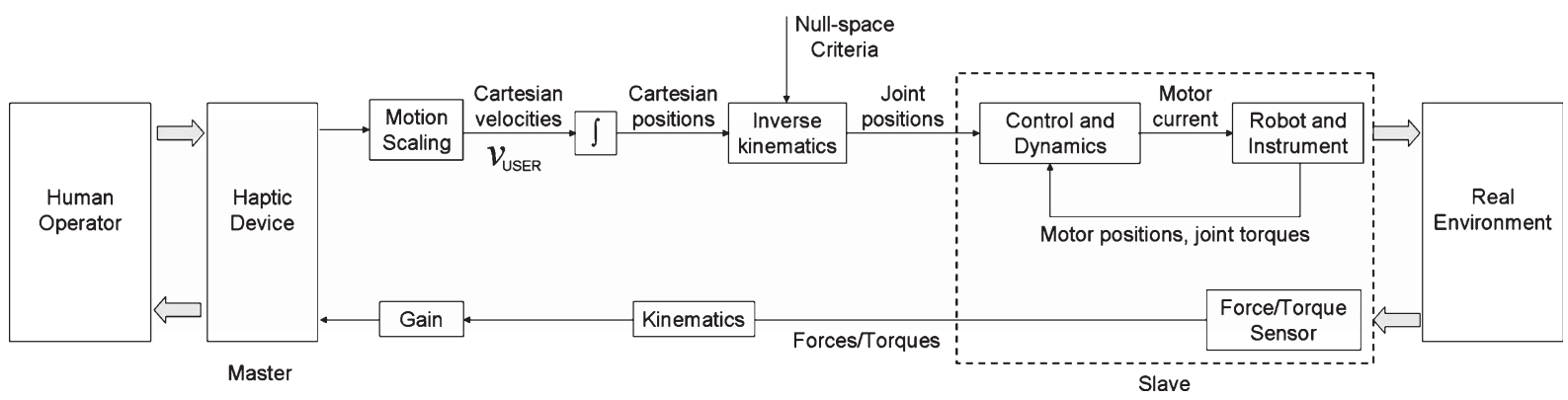

Fig. 2. Basic scheme of force feedback telemanipulation for the MiroSurge platform.

desired joint angles are sent to the slave's controller which takes care of fast motion tracking. For the control of the MIRO robots, a full-state feedback control for flexible and coupled joints is implemented [25]. The controlled states are the motor positions and joint torques which are both directly measured, as well as the derivatives of both measurements. An additional disturbance observer uses the joint torque measurements to reduce friction in the motor and the gear boxes [26].

Motion compensation. To integrate motion compensation, the target motion is captured by a stereo camera and fed to the heart tracking module as shown in Fig. 3. The heart tracking module has to generate as output the Cartesian velocity of an ROI $v_{\mathrm{ROI}}$, written in the coor- dinate system (COS) of the camera. This velocity is then transformed into the COS of the instrument tool center point (TCP). Depending on whether the motion compensation is turned on $(H=1)$ or off $(H=0)$, the tracked velocity is added to the Cartesian velocity $v_{\text {USER }}$ coming from the input device.

Online soft tissue simulation. The user has the option to either interact with the real organ or with the simulation of the organ, see switch $S$ in Fig. 3. In both cases force feedback is available. In case the user interacts with the real scenario $(S=0)$, the simulation is running in parallel and provides monitoring. This way, large discrepancies between the measured real interaction forces and the forces calculated in the simulation may be used as indicator for sensor mal-

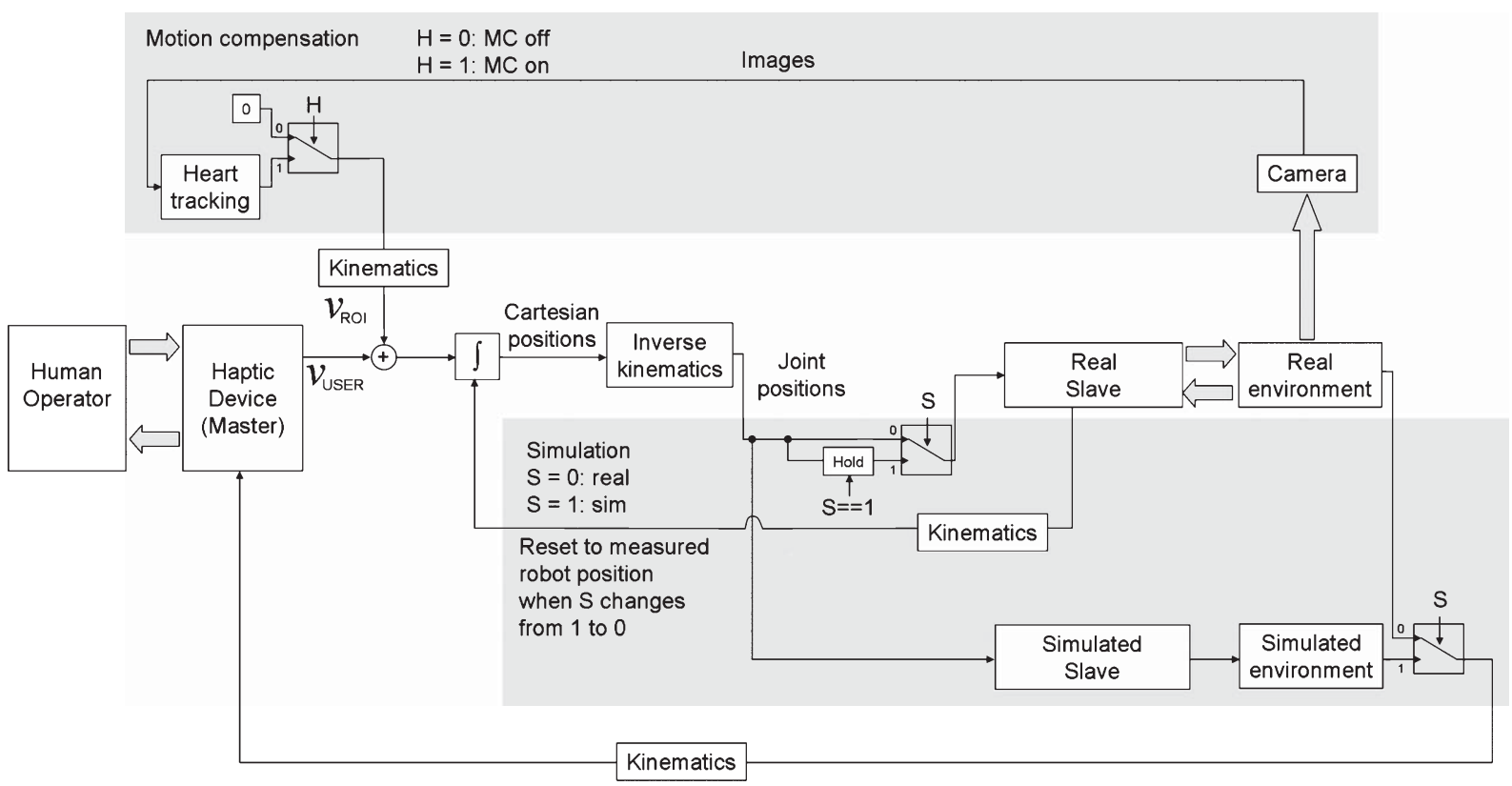

Fig. 3. Integration of the additional features motion compensation and online soft tissue simulation into the Mirosurge control scheme. 
function or bad registration. If the user is interacting with the simulation $(S=1)$, the robotic system is not moving. When switching back from the simulation to real interaction, the integrator has to be reset to the actual instrument TCP. For simulation, the simulated slave is idealized by simple forward kinematics, thus assuming that the virtual robot instantaneously reacts to desired motions. In future work, this can be replaced by a more complex physical model of the robotic system.

\subsection{Tracking the beating heart}

Due to the complex nature of the heart surface, a suitable choice for a deformation model is complicated as shown in Sec. 1. The parametric models currently used to describe the heart surface display problems with specular reflections and textureless (homogeneous) regions. For instance, the B-spline mapping in [24] does not employ any surface regularization, which may lead to erroneous shape estimates in regions without sufficient texture. Similarly, the bilinear mapping in [43] needs a very large number of control points to sufficiently approximate the heart surface shape.

For such reasons, a type of Radial Basis function called the Thin-Plate Splines (TPS) for modeling the heart surface deformations is chosen. The TPS was successfully applied for modeling non-rigid tissue deformation $[4,3,28]$. One of the most interesting features of the TPS mapping is the flexibility in placing its control points. This represents the key feature that allows to overcome the difficulties of previous methods concerning the lack of visual information when tracking regions with poor texture.

Based on extensions to the original TPS affine formulation $[32,4]$ a region-based method for tracking the heart surface deformations with a novel parameterization for 3D tracking in the stereo scenario using the TPS was developed [38]. This parameterization offers an efficient formulation of the tracking problem, without requiring disparity search or other intermediary steps that may reduce the tracking accuracy.

Initially, a smooth and continuous region of interest on the heart surface is selected as the reference image T. Next, to model the heart surface deformation, a Thin-Plate Spline parametric model is used. The TPS is a Radial Basis Function characterized by the basis function $U(s)=s^{2} \log \left(s^{2}\right),(n+3)$ parameters $\left(w_{1}, \ldots, w_{\mathrm{n}}, r_{1}, r_{2}, r_{3}\right)^{T}$ and a set of $\mathrm{n}$ control points $\mathbf{c}_{i}=\left(\hat{x}_{i}, \hat{y}_{i}\right)$, such that a spatial mapping of pixels $\mathbf{x} \mapsto f$ $(\mathbf{x})$ is calculated as:

$$
f(\mathbf{x})=r_{1}+r_{2} x+r_{3} y+\sum_{i=1}^{n} w_{i} U\left(\left\|\mathbf{c}_{i}-\mathbf{x}\right\|\right)
$$

where $\|\cdot\|$ denotes the Euclidean norm. The control points define the degrees of freedom of the warping model (more or less control points can be used to account for the local heart surface deformation).

In the monocular case, two TPS functions $f^{\mathrm{x}}$ and $f^{\mathrm{y}}$ sharing their control points define a mapping of the pixel positions of the selected reference image onto the current image of the operating field. For tracking in $3 \mathrm{D}$, we seek to align the same reference image on both stereo images simultaneously. Consequently, a TPS warping $w(\mathbf{x}, \mathbf{h}, \mathbf{C})$ of a pixel $\mathbf{x}$ can be defined as function of a vector $\mathbf{h}$ of Cartesian coordinates of points in space that map to the control points on each stereo image and the respective camera matrix $\mathbf{C}$ (for more details, see [38]).

Consequently, the 3D tracking problem consists in estimating the optimal warping parameter vector $\mathbf{h}$ that minimizes the alignment error $\varepsilon$ between the reference image $\mathbf{T}$ and both left and right images of the stereo pair $\mathbf{I}_{l}$ and $\mathbf{I}_{r}$ simultaneously:

$$
\begin{aligned}
\min _{\mathrm{h}} \varepsilon= & \sum_{\mathbf{x} \in \mathbf{A}}\left[\left[\mathbf{I}_{l}\left(w\left(\mathbf{x}, \mathbf{h}, \mathbf{C}_{l}\right)\right)-\mathbf{T}(\mathbf{x})\right]^{2}\right. \\
& \left.+\left[\mathbf{I} r\left(w\left(\mathbf{x}, \mathbf{h}, \mathbf{C}_{r}\right)\right)-\mathbf{T}(\mathbf{x})\right]^{2}\right]
\end{aligned}
$$

where $\mathrm{A}$ is the set of the template coordinates and $\mathbf{I}_{1}(w(\mathbf{x}, \mathbf{h}, \mathbf{C}))$ and $\mathbf{I}_{\mathbf{r}}(w(\mathbf{x}, \mathbf{h}, \mathbf{C}))$ are the current left or right images transformed by the warping function $w(\mathbf{x}, \mathbf{h}, \mathbf{C})$, respectively.

For solving the minimization problem above, we use the efficient second-order minimization (ESM) algorithm proposed by [7]. The ESM is applied because it displays a faster convergence rate and larger convergence basin than traditional optimization techniques such as Gauss-Newton or Levenberg-Marquardt. In addition, for increasing the robustness towards illumination variations the illumination compensation method proposed by Silveira et al. [8] is incorporated to the tracking framework. Tracking results obtained with the proposed technique on an in vivo beating heart are illustrated in figure 4 . A schematic overview of the tracking algorithm is given in Fig. 5.

Implementation on a Graphics Processor Unit $(G P U)$. The visual tracking algorithm must extract 

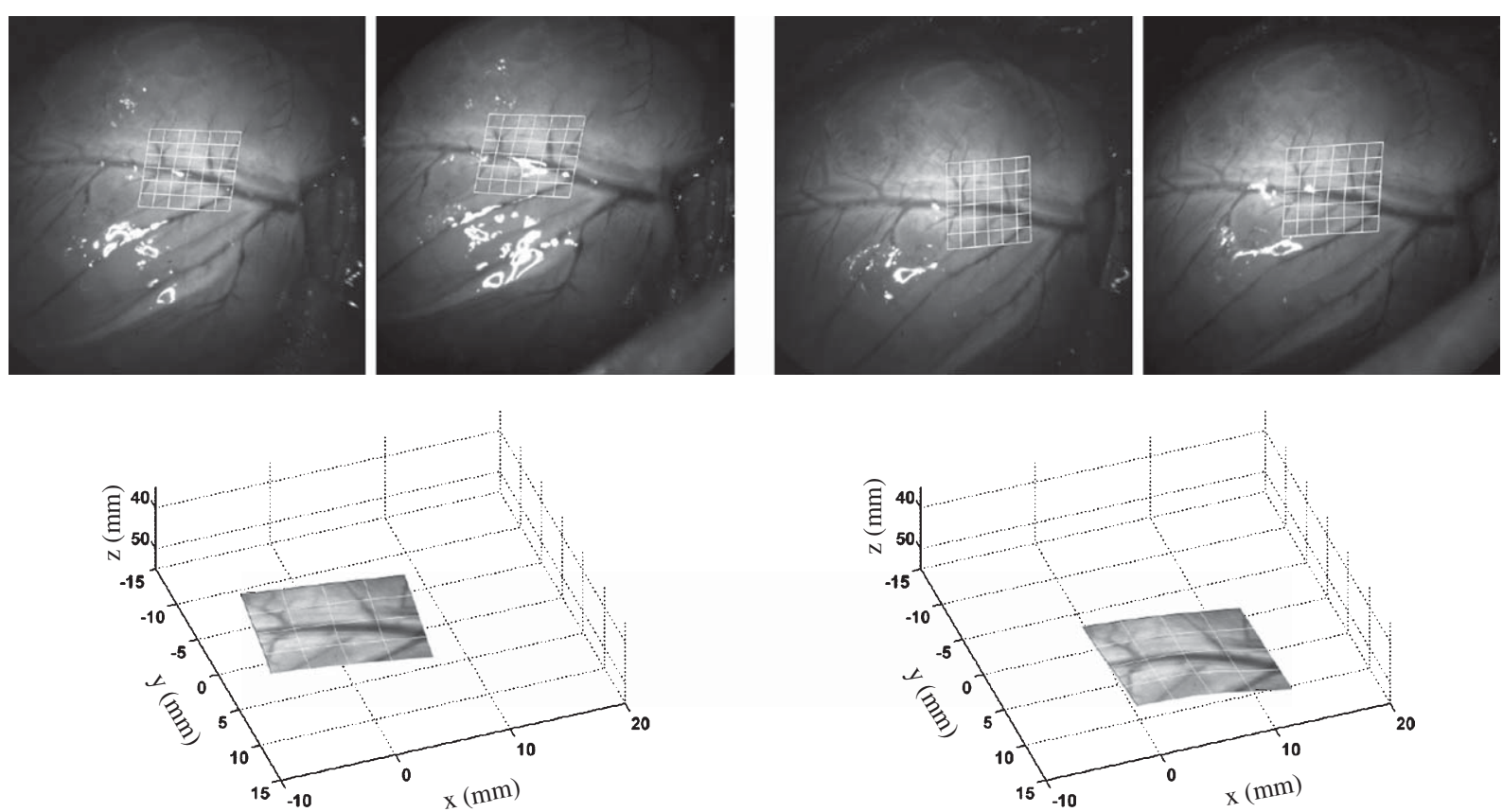

Fig. 4. Endoscopic stereo images of a porcine beating heart, acquired at high speed $(83 \mathrm{~Hz})$ with a very small baseline [39]. Notice the difficulties involved in acquiring at high speeds due to insufficient lighting -For different instants of the heart cycle, the target region tracked on the left and right images of the stereo pair and the corresponding TPS surface approximation are illustrated. The reconstruction uses the left camera as world coordinate frame. The tracking algorithm is robust to illumination variations and specular reflections.

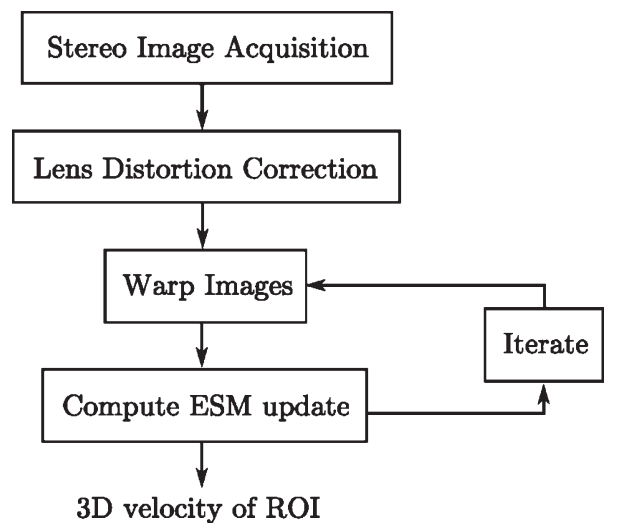

Fig. 5. A schematic overview of the tracking algorithm.

the heart motion on-line for an accurate synchronization with the robotic tools. This implies that tracking must run at high frame-rates. The first efforts towards a fast implementation of the tracking algorithm were far below the desired tracking frame-rate $(\approx 5 \mathrm{~Hz}$ in an implementation using Intel Performance Primitives -IPP). Due to the larger computational requirements of the application, the computational power of recently released GPUs is explored.

The market for real-time high-definition 3D graphics has given birth to high-parallel, multi-core processors with great computation power. Since GPUs are specially well-suited to address problems that can be expressed as data-parallel computations, an implementation of the tracking algorithm was developed in order to take advantage of the hardware efficient processing. For the experiments, a NVidia GTX280 (Santa Clara, EUA) graphics card programmed using CUDA (a programming extension to $\mathrm{C}$ ) was used.

The computation time per iteration may vary depending on the size of the reference image, the number of spatial and illumination parameters. For instance, considering a $128 \times 128$ pixel region of interest with 5 control points the computation time per iteration required for tracking is of $\approx 2.25 \mathrm{~ms}$. Assuming a transfer delay of around $0.2 \mathrm{~ms}$ from the cameras to the graphics card memory, tracking speeds over $50 \mathrm{~Hz}$ can be achieved $(\mathrm{a} \times 10$ speed improvement in comparison with the CPU implementation). It is noticeable that the number of iterations required for the tracking algorithm to converge depends on the inter- 
frame motion, which is directly related to the image acquisition speed. If the acquisition speed is increased, the heart displacement between frames is reduced and less iterations are necessary for tracking to converge. Therefore, the computational requirement per frame decreases with an increase of the acquisition speed.

\subsection{Soft tissue modelling}

The workflow followed to provide online simulation of human organs starts from the CT acquisition of the patient. In the scope of this paper, a silicone model of a liver with stiffer inclusions is used (see Sec. 3). The subsequent steps are CT image segmentation, MSM generation and, finally, the actual simulation. During the whole process a rigid structure firmly attached to the model provides a common frame of reference for the different phases.

Following the semi-automatic procedure described in [10], the liver and the inclusions is segmented. To obtain the segmentation three steps are applied: liver segmentation using a watershed based algorithm, optimal inclusion threshold identification by entropy minimization and segmentation refinement with hole filling and surface smoothing. The overall procedure requires some manual tuning of parameters due to the differences in Hounsfield values between real tissues and synthetic phantom.

The different volumes (liver and inclusions) identified by the segmentation phase are discretized into a single tetrahedral mesh. A label is associated to each tetrahedra to keep track of the constitutive material (i.e. liver parenchyma, tumors, ...). From this mesh the $M S M$ structure is simply obtained by placing a mass point in each vertex of the mesh and a spring-damper system in correspondence of each mesh edge. Tetrahedra labels and original CT data are used during the subsequent calibration phase. In the specific case of the synthethic model the imporant parameters of the different material needed for its realization are identified: density and stiffness. These parameters are used with a calibration method based on the work proposed in [51] to associate a mass value to each model vertex and elastic and damping coefficients to each model spring. Key features of the obtained model can be found in Table 1, and the simulated organ is shown in Fig. 6.

In order to achieve a registration of the virtual organ with the real one, a surface scan of the organ is performed with the $3 \mathrm{D}-$ Modeller (3DMo). The $3 \mathrm{DMo}$ is a handheld device for capturing the surface of objects.
Table 1

Key features of the synthetic liver model

\begin{tabular}{|c|c|c|c|c|c|}
\hline \multicolumn{4}{|c|}{ Complete Mesh } & \multirow{2}{*}{$\begin{array}{c}\text { Liver } \\
\text { Density }\end{array}$} & \multirow{2}{*}{$\begin{array}{l}\text { Inclusion } \\
\text { Elasticity }\end{array}$} \\
\hline es & Springs & Density & & & \\
\hline 16398 & 90592 & $1326 \mathrm{Kg} / \mathrm{m}^{3}$ & $U k P a$ & $1326 \mathrm{Kg} \mathrm{f} / \mathrm{m}^{5}$ & $31 \mathrm{kPa}$ \\
\hline
\end{tabular}

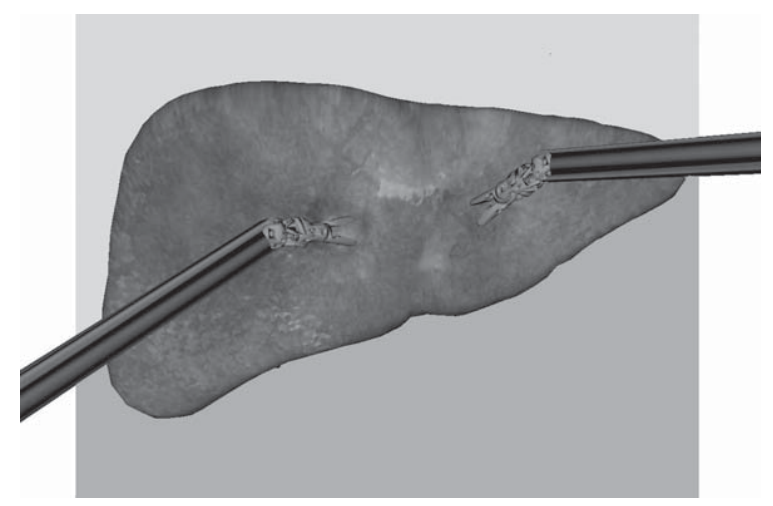

Fig. 6. The synthetic liver model being manipulated by virtual robots.

The generated mesh is then matched with the virtual organ surface automatically [22]. The method was developed for open surgery. In a minimally invasive surgery the organs are not accessible to the 3DMo due to the size of the device, therefore new algorithms that allow to replace the 3DMo by a stereo camera system are currently adopted.

The last phase of this process is the online simulation of the obtained deformable model. The simulation runs along with the reality and mimics the effects of surgeon action on the real models, the realism of the simulation allows the user to seamlessy switch between real and virtual environment.

To exploit GPU computational power data is encoded in a format that allows parallel processing and that follows the limitations imposed by GPU memory management. The presented simulator is based and extends the work proposed in [1] to which the reader is referred. Each simulation step is composed by three phases: collision detection and solution, internal force computation, temporal integration of model configuration. All those phases are carried out by the $G P U$, the only data exchanged with $C P U$ are the virtual tool position and the resulting interaction force.

The use of the GPU allows the physical simulation to run at more than $1 \mathrm{kHz}$ and the graphics of the scene to be rendered at $30 f \mathrm{ps}$. This ensures the realism of both haptic and graphic feedback. 

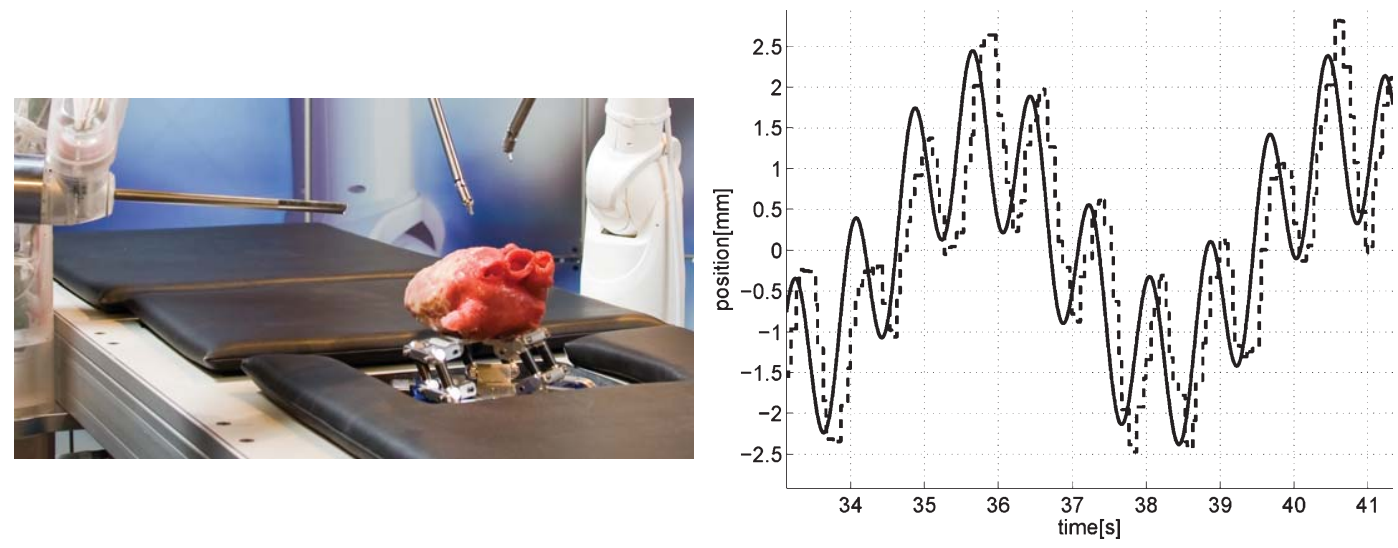

Fig. 7. Heart motion simulator (left) and plot of the desired motion of the heart simulator and the tracked motion (dashed), (right).

\section{Preliminary experimental validations}

A custom made silicone model of the heart is used as target for tracking. The heart model is mounted to a parallel robot that allows for translational motions. As target motion, a dual sinus in the image plane of the endoscope is assumed. It models the heart contractions with frequency $f_{\text {heartbeat }}=1.25 \mathrm{~Hz}$, amplitude $a_{\text {heartbeat }}=1 \mathrm{~mm}$ as well as the breathing with $f_{\text {breathing }}=0.2 \mathrm{~Hz}$ and amplitude $a_{\text {breathing }}=1.4 \mathrm{~mm}$. Harmonics of these base frequencies are not included, and there is a zero offset between the two sinusses. Despite these simplifications, the resulting motion resembles a real heart motion as shown e.g. in [46, 39]. Figure 7 (left) shows the heart motion simulator. The heart motion is captured by a stereo endoscope with $25 \mathrm{~Hz}$.

To evaluate the online soft tissue modelling, a silicone model of a liver with included tumors was built, see Fig. 8. The physical parameters for healthy tissue and for tumors are taken from literature. Both organs are placed according to the human anatomy beneath a transparent replication of a human torso, see Fig. 9 (right).

Motion compensation. The desired motion of the heart simulator and the tracked motion of the ROI are shown in Fig. 7 (right). The motion tracking reliably computes the velocities of the ROI in less than $120 \mathrm{~ms}$. The motion is then fed to the robot control to achieve the motion compensation behaviour of the robot. Since no motion prediction is included, a slight delay between the desired and tracked motion can be seen in Fig. 7 (right).

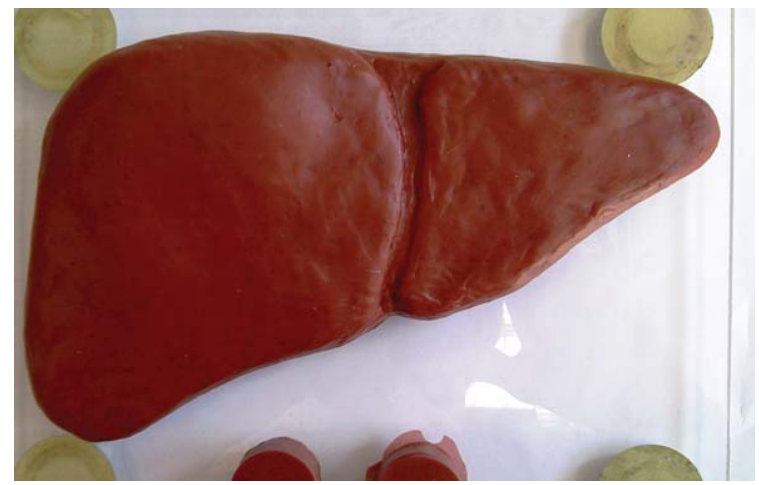

Fig. 8. Silicone liver model.

The delay between the image acquisition and visual tracking step to the robot actuation can be compensated by exploiting the quasi-periodicity of the beating heart motion for predicting its future motion. Although no motion prediction is incorporated to the current system, studies show that low prediction errors can be achieved in small prediction horizons (see [40] such as the measured compensation delay $(120 \mathrm{~ms})$.

Online soft tissue modelling. The described setup proved that seamless switching between the real and the simulated environment is possible and useful. A qualitative comparison between contact forces showed that tumor localization was possible both in the real and simulated environment. The findings are consistent with results from manual palpation. Figures 9 and 10 show the integrated setup. The integration of the interactive, physically based simulator in the architec- 

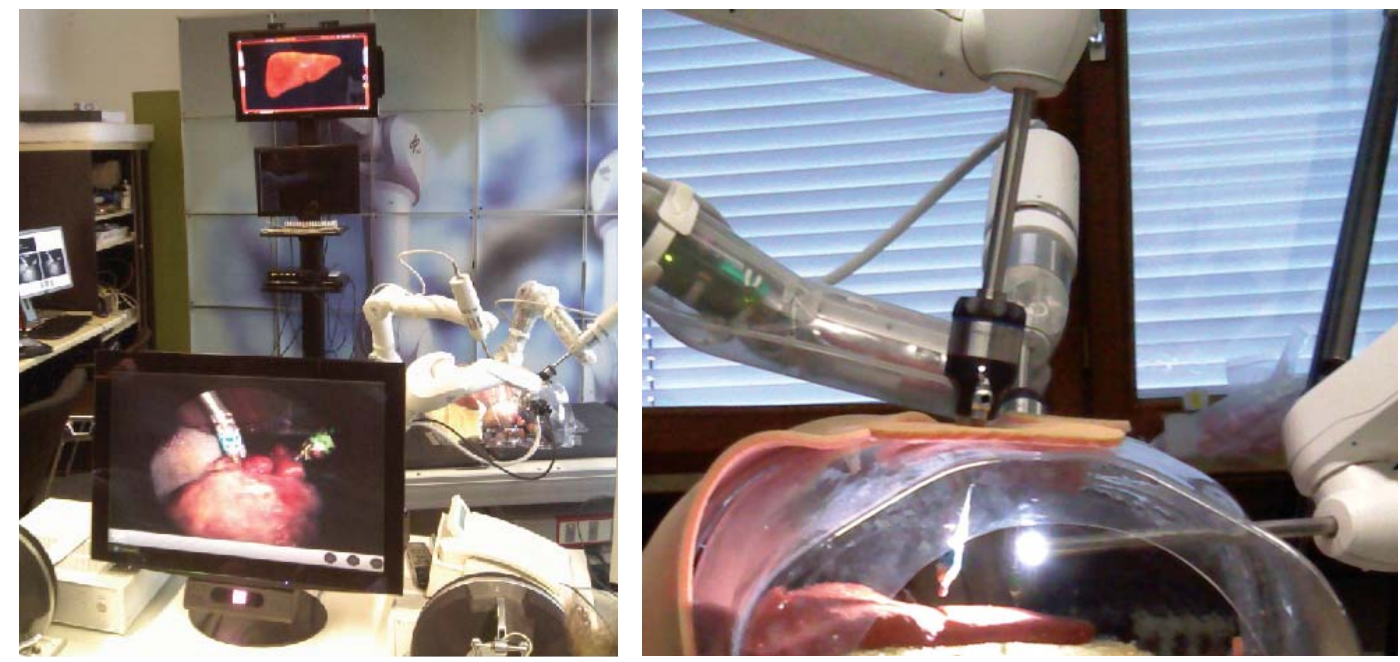

Fig. 9. Integration of the additional feature online soft tissue simulation into the MiroSurge platform. On the left the screen arrangement is shown: The front screen provides the actual image from the real stereo endoscope, while the screen on top provides a rendered scene. The slave side of the MiroSurge system during a palpation task is shown on the right.

ture provides some benefits to the overall procedure. During our tests the simulator received the input from the master controllers and from the camera controller. Physics simulation was updated at a frequency of more than $1200 \mathrm{~Hz}$ with a synthetic liver model composed by more than 16,000 mass points and about 90000 spring-damper systems. Subjective evaluation of haptic feedback provided by surgeons proved that the deformable model response is comparable with the real one. Objective validation of simulated data in realistic environment will require more advanced techniques and should be performed on real biologic tissues.

During the described test simulator efficiently provides three enhancements to the interventional scenario. It allows the surgeon to control a virtual camera in free motion inside the scene, to obtain a clearer view of the interventional area and to better understand the relative position of tools and organ of interest. The addition of transparencies to the rendered scene allows the user to see the inner structures of the organs. In the specific case (see Fig. 10, right), it is possible to display the inclusions of the synthetic liver, thus guiding the user during the intervention. Last, the developed architecture allows the user to switch between the real scenario and the virtual one, to try some actions in simulation before performing them in reality. Results prove that the simulation can run along with the reality, thus satisfying required real time constraints, moreover the simulation correctly mimics the reality in both graphic and haptic rendering.
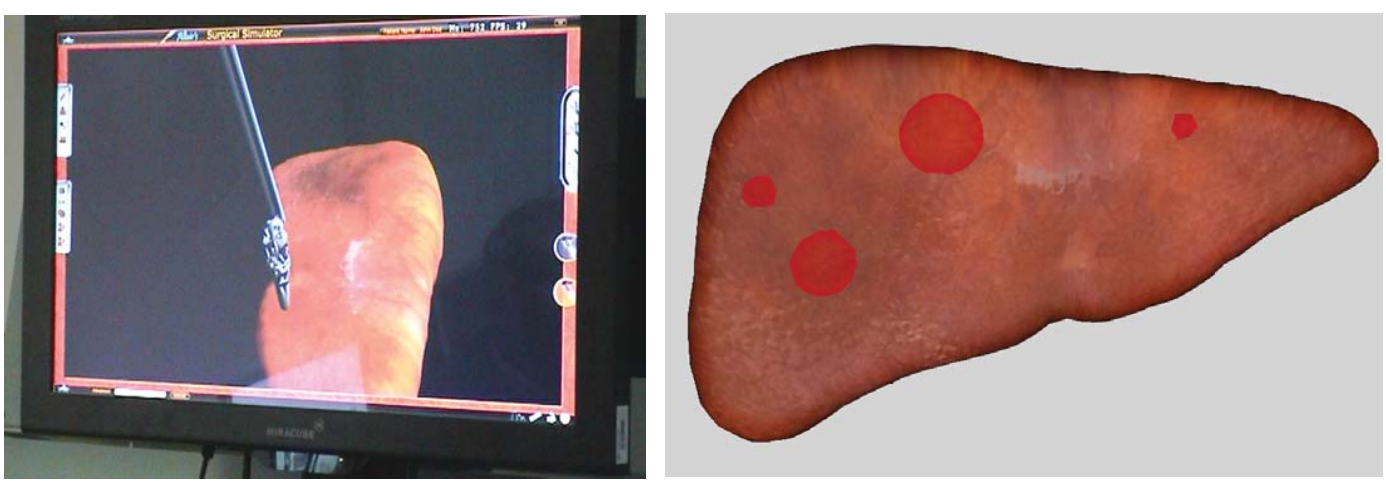

Fig. 10. Rendered scene while monitoring the motion of the robotic tool (left) and transparent rendering to show the inclusions inside the liver (right). 


\section{Conclusion}

New modules for motion compensation for beating heart surgery and online soft tissue modelling were successfully integrated into the Mirosurge platform.

Motion compensation is an important feature to increase surgeon accuracy when operating on moving organs. To the authors' knowledge, this is the first vision-based 3D motion compensation system to incorporate a surgical platform. This paper shows the feasibility of its integration into a complex telemanipulation system in $6 \mathrm{DoF}$ with dedicated kinematics for the entry point into the patient. To further enhance the quality of the motion compensation, a higher image resolution of the endoscope would be beneficial for capturing the details of fine heart structures. Another important issue is the processing delay which includes the image acquisition by the endoscope, tracking, control and the actuation stages. If this delay is not sufficiently low, critical errors can be induced in the motion compensation system. Even though palliative solutions such as motion prediction can compensate for this delay in most of the cases, the compensation errors due to latency should be kept within safe levels (under $200 \mu \mathrm{m}$ ) required by cardiac surgery [9]. In fact, recent studies show that low prediction errors can be achieved in small prediction horizons (see [40]) such as the measured compensation delay $(120 \mathrm{~ms})$. The evaluation of motion prediction methods (as developed e.g in. [36]) with the MiroSurge platform is future work. These methods could also be used to bridge situations when the view of the camera to the ROI is occluded by the instruments. In addition, once the instruments are in contact with the organ, an approach using the measurement of contact forces as well as vision for motion compensation may be advantageous. To provide the user with a view on the virtually still standing organ, the velocities generated from the motion tracking also need to be fed into the visualization at the master console.

The accurate estimation of the deformations of internal organs as provided by the tracking algorithm is also a key requirement for merging pre-operative and intraoperative data. Patient-specific information such as the internal organ structures can be used for increased surgical safety and surgical guidance once precise information about the online poses of the organ is available.

Online soft tissue modeling in the MiroSurge scenario has shown potential to assist the user during operation. The innovations introduced by our approach are manifold: the physical modeling of the scene provides, in fact, realistic reconstruction of the interventional area that allows to assist the surgeons in effective ways. In particular the surgeon can freely navigate inside the scene, thus overcoming endoscope motion constraints. The surgeon can also exploit advanced rendering of the scene, that provides the view of hidden structures or inclusions such as tumors, vessels or ducts. The last advantage provided by the proposed method is the ability to switch between the real environment and the virtual one. This allows the user to explore the area or to try some parts of the intervention without risk for the patient, thus providing a better understanding of the ongoing surgery.

A crucial challenge remains to achieve online registration of the organ pose and of its physical properties, especially in case the organ is changed during operation e.g. when removing a tumor. The possibility to solve this with a motion tracking algorithm such as the one presented remains to be analyzed. In the case of pure palpation, the online modeling already now provides valuable information.

The integration of the presented modules into the MiroSurge platform was possible without major changes in the existing control schemes presented in [47]. The achieved modularity facilitates e.g. comparisons of the presented motion tracking algorithm with alternative approaches as presented in [15] Thus, the MiroSurge system provides a valuable platform for the development and evaluation of new advanced features in robotic telesurgery.

\section{Acknowledgments}

This work is supported by the AccuRobAs project under the 6th Framework Programme (FP6) of the European Union.

\section{References}

[1] M. Altomonte, D. Zerbato, D. Botturi and P. Fiorini, Simulation of deformable environment with haptic feedback on gpu, in Intelligent Robots and Systems, 2008. IROS 2008. IEEE/RSJ International Conference on, 2008, pp. 3959-3964.

[2] T. Bader, A. Wiedermann, K. Roberts and U.D. Hanebeck, Model-based motion estimation of elastic surfaces for mini-mally invasive cardiac surgery, in Proceedings of IEEE Conference on Intelligent Robots and Systems IROS 07, San Diego, USA, 2007, pp. 871-876. 
[3] A. Bartoli and A. Zisserman, Direct estimation of non-rigid registrations, in Proceedings of the 15th British Machine Vision Conference, vol. 2, Kingston, UK, 2004, pp. 899-908.

[4] A. Bartoli, M. Perriollat and S. Chambom, Generalized thinplate spline warps, in Proceedings of IEEE Conference on Computer Vision and Pattern Recognition (CVPR 07), Minneapolis, USA, 2007, pp. 1-8.

[5] C. Basdogan, M. Sedef, M. Harders and S. Wesarg, VR-based simulators for training in minimally invasive surgery, IEEE Computer Graphics and Applications 27 (2007), 54-66, ISSN 0272-1716.

[6] K. Bathe. Finite Element Procedures, Prentice Hall, 1995, ISBN 9780133014587

[7] S. Benhimane and E. Malis, Real-time image-based tracking of planes using efficient second-order minimization, in Proceedings of IEEE Conference on Intelligent Robots and Systems IROS '04, Sendai, Japan, 2004, pp. 943-948.

[8] S. Benhimane and E. Malis, Homography-based 2d visual tracking and servoing, International Journal of Computer Vision 267 (2007), 661-676.

[9] C. Cavusoglu, J. Rotella, W.S. Newman, S. Choi, J. Ustin and S.S. Sastry, Control algorithms for active relative motion cancelling for robotic assisted off-pump coronary artery bypass graft surgery, In Proceedings of the 12th International Conference on Advanced Robotics (ICAR), Seattle, USA, 2005, pp. 431-436.

[10] Anirudh Choudhary, N. Moretto, F. Pizzorni Ferrarese and G.A. Zamboni, An entropy based multi-thresholding method for semi-automatic segmentation of liver tumors, The MIDAS Journal -Grand Challenge Liver Tumor Segmentation 2008 MICCAI Workshop, 2008.

[11] Force Dimension, The omega haptic master device, [web page], 2010. URL http://www.forcedimension.com. [Accessed on February 2nd, 2010].

[12] Y.C. Fung, Biomechanics -Mechanical Properties of Living Tissues, Springer-Verlag, second edition, 1993.

[13] J. Georgii, F. Echtler and R. Westermann, Interactive simulation of deformable bodies on GPUs, in In SimVis 05, SCS Publishing House e.V, 2005, pp. 247-258.

[14] M. Gröger, W. Sepp, T. Ortmaier and G. Hirzinger, Reconstruction of image structure in presence of specular reflections, in B. Radig and S. Florczyk, editors, Pattern Recognition, Proc. 23rd DAGM Symposium, volume 2191 of LNCS, Munich, Germany, Springer, 2001, pp. 53-60.

[15] M. Gröger, T. Ortmaier, W. Sepp and H. Gerd, Tracking local motion on the beating heart, in SPIE medical imaging, editor, Visualization, Image-Guided Procedures and Display, volume 4681, SPIE, 2002, pp. 233-241.

[16] U. Hagn, T. Ortmaier, R. Konietschke, B. Kübler, U. Seibold, A. Tobergte, M. Nickl, S. Jörg and G. Hirzinger, Tele-manipulator for remote minimally invasive surgery, IEEE Robotics and Automation Magazine 154 (2008), 28-38, ISSN 1070-9932.

[17] U. Hagn, R. Konietschke, A. Tobergte, M. Nickl, S. Jörg, G. Passig, M. Gröger, U. Seibold, L. Le-Tien, S. Jörg, B. Kübler, F. Fröhlich, A. Albu-Schäffer, A. Nothhelfer, F. Hacker, M. Grebenstein and G. Hirzinger, DLR MiroSurge: a versatile system for research in endoscopic telesurgery, International Journal of Computer Assisted Radiology and Surgery, 5 (2009), 183-193.

[18] James and D.K. Pai, Dyrt: dynamic response textures for real time deformation simulation with graphics hardware, in $S I G$ -
GRAPH'02: Proceedings of the 29th annual conference on Computer graphics and interactive techniques, New York, NY, USA, ACM, pp. 582-585, ISBN 1-58113-521-1 2002.

[19] Keiser, B. Adams, D. Gasser, P. Bazzi, P. Dutre and M. Gross, A unified lagrangian approach to solid-fluid animation, In In Symposium on Point-Based Graphics, Eurographics Association, 2005, pp. 125-133.

[20] R.M. Koch, M.H. Gross, F.R. Carls, D.F. von Büren, G. Fankhauser and Y.I.H. Parish, Simulating facia surgery using finite element models, Computer Graphics, 30 (Annual Conference Series), 1996, 421-428, URL citeseer.ist.psu.edu/koch96simulating.html.

[21] R. Konietschke, Planning of Workplaces with Multiple Kinematically Redundant Robots, Technische Universität München, PhD Thesis, Munich, Germany, 2007.

[22] R. Konietschke, A. Busam, T. Bodenmüller, T. Ortmaier, M. Suppa, J. Wiechnik, T. Welzel, G. Eggers, G. Hirzinger and R. Marmulla, Accuracy Identification of Markerless Registration with the DLR 3D-Modeller in Medical Applications, in 6. Jahrestagung der Deutschen Gesellschaft für Computer-und Roboterassistierte Chirurgie CURAC, Karlsruhe, Germany, 2007, 11-13.

[23] N. Langrana, G. Burdea, J. Ladeji and M. Dinsmore, Human performance using virtual reality tumor palpation simulation, Computers \& Graphics, Haptic Displays in Virtual Environments and Computer Graphics in Korea, 214, 1997, 451-458, ISSN 0097-8493.

[24] W. Lau, N.A. Ramey, J.J. Corso, N.V. Thakor and G.D. Hager, Stereo-based endoscopic tracking of cardiac surface deformation, In Medical Image Computing and Computer-Assisted Intervention (MICCAI'04), volume 3217 of Lecture Notes in Computer Science (LNCS), Saint Malo, France, Springer 2004, pp. 494-501

[25] L. Le-Tien, A. Albu-Schäffer and G. Hirzinger, MIMO state feedback controller for a flexible joint robot with strong joint coupling, in Proceedings of the IEEE International Conference on Robotics and Automation ICRA, Roma, Italy, DOI 10.1109/ROBOT.2007.364065, 2007, pp. 3824-30.

[26] L. Le-Tien, A. Albu-Schäffer, A. De Luca and G. Hirzinger, Friction observer and compensation for control of robots with joint torque measurement, in IROS, 2008, pp. 3789-3795.

[27] Q. Li and K.-M. Lee, An adaptive meshless method for modeling large mechanical deformation and contacts, Robotics and Automation, 2007 IEEE International Conference on, pp. 1207-1212, ISSN 1050-4729 2007.

[28] J. Lim and M. Yang, A direct method for modeling non-rigid motion with thin plate spline. In Proceedings of IEEE Conference on Computer Vision and Pattern Recognition CVPR '05, Washington, USA, 2005, pp. 1196-1202.

[29] Y.-J. Lim, J. Hu, C.-Y. Chang and N. Tardella, Soft tissue deformation and cutting simulation for the multimodal surgery training, In CBMS'06: Proceedings of the 19th IEEE Symposium on Computer-Based Medical Systems, Washington, DC, USA, IEEE Computer Society, 2006, pp. 635-640, ISBN 0-7695-2517-1.

[30] B. Lo, A.J. Chung, D. Stoyanov, G. Mylonas and G-Z Yang, Real-time intra-operative $3 \mathrm{~d}$ tissue deformation recovery, In Proceedings of IEEE International Symposium on Biomedical Imaging ISBI 08, Paris, France, 2008a, pp. 1387-1390.

[31] B. Lo, M. Scarzanella, D. Stoyanov and G. Yang, Belief propagation for depth cue fusion in minimally invasive surgery, in Medical Image Computing and Computer-Assisted Interven- 
tion MICCAI'08, Lecture Notes in Computer Science LNCS, New York, USA, Springer, 2008, pp. 104-112.

[32] E. Malis, An efficient unified approach to direct visual tracking of rigid and deformable surfaces, in Proceedings of IEEE Conference on Intelligent Robots and Systems IROS '07, San Diego, USA, 2007, pp. 2729-2734.

[33] J. Mosegaard, P. Herborg and T.S. Sørensen, A gpu accelerated spring mass system for surgical simulation, in Medicine Meets Virtual Reality 13: The Magical Next Becomes the Medical Now, 2005, pp. 342-348.

[34] M. Müller, B. Solenthaler, R. Keiser and M. Gross, Particlebased fluid-fluid interaction, in SCA '05: Proceedings of the 2005 ACM SIGGRAPH/Eurographics symposium on Computer animation, New York, NY, USA, ACM, 2005, pp. 237-244, ISBN 1-7695-2270-X.

[35] D.G. Murphy, B.J. Challacombe and A.J. Costello, Outcomes After Robot-assisted Laparoscopic Radical Prostatectomy, Asian Journal of Andrology 11 (2009), 94-99.

[36] T. Ortmaier, M. Gröger, D.H. Boehm, V. Falk and G. Hirzinger, Motion estimation in beating heart surgery, IEEE Transactions on Biomedical Engineering 5210 (2005), 1729-1740.

[37] R. Osborne, J. Samosky, A. Sawada, S. Gibson, C. Fyock, E. Grimson, T. Kanade, R. Kikinis, H. Lauer, N. Mckenzie, A. Mor, S. Nakajima and H. Ohkami, Volumetric object modeling for surgical simulation, Medical Image Analysis 2 (1998), 121-132.

[38] R. Richa, P. Poignet and C. Liu, 3D motion tracking for beating heart surgery, International Journal of Robotics Research 29(2-3) (2010), 218-230.

[39] R. Richa, A. Bo and P. Poignet, Beating heart motion prediction for robust visual tracking, ICRA'10: 2010 IEEE International Conference on Robotics and Automation, USA, 2010, pp. 4579-4584.

[40] R. Richa, A. Bo and P. Poignet, Towards Robust 3D Visual Tracking For Motion Compensation in Beating Heart Surgery, Medical Imaging Analysis 15(3) (2011), 302-315.

[41] G. Silveira and E. Malis, Real-time visual tracking under arbitrary illumination changes, in Proceedings of IEEE Confer-ence on Computer Vision and Pattern Recognition CVPR'07, Minneapolis, USA, 2007, pp. 1-6.

[42] D. Stoyanov and G.Z. Yang, Stabilization of image motion for robotic assisted beating heart surgery, in Medical Image Computing and Computer-Assisted Intervention (MICCAI'07), volume 4791 of Lecture Notes in Computer Science (LNCS), Brisbane, Australia, Springer, 2007, pp. 417-424.

[43] D. Stoyanov, A. Darzi and G. Yang, A practical approach towards accurate dense 3D depth recovery for robotic laparo-scopic surgery, Computer Aided Surgery, 410 2005a, 199-208.

[44] D. Stoyanov, G.P. Mylonas, F. Deligianni, A. Darzi and G.Z. Yang, Soft-tissue motion tracking and structure estimation for robotic assisted mis procedures, In Medical Image Computing and Computer-Assisted Intervention MICCAI'05, volume 3750 of Lecture Notes in Computer Science (LNCS), Palm Springs, USA, Springer, 2005b, pp. 139-146.

[45] R.H. Taylor and D. Stoianovici, Medical Robotics in Computer-Integrated Surgery, IEEE Transactions on Robotics and Automation 195 (2003), 765-781.

[46] A. Thakral, J. Wallace, D. Tomlin, N. Seth and N.V. Thakor, Surgical motion adaptive robotic technology s.m.a.r.t: Taking the motion out of physiological motion, In Medical Image Computing and Computer-Assisted Intervention (MICCAI'01), Lecture Notes in Computer Science (LNCS), Utrecht, The Netherlands, Springer, 2001, pp. 317-325.

[47] A. Tobergte, R. Konietschke and G. Hirzinger, Planning and control of a teleoperation system for research in minimally invasive robotic surgery, in ICRA, 2009, pp. 4225-4232.

[48] A.L. Trejos, S.E. Salcudean, F. Sassani and S. Lichtenstein, On the feasibility of a moving support for surgery on the beating heart, in Medical Image Computing and Computer-Assisted Intervention (MICCAI'99), volume 1679 of Lecture Notes in Computer Science (LNCS), Cambridge, UK, Springer, 1999, pp. 1088-1098.

[49] M. Visentini-Scarzanella, G.P. Mylonas, D. Stoyanov and G. Yang, $i$-brush: A gaze-contigent virtual paintbrush for dense $3 \mathrm{~d}$ reconstruction in robotic assisted surgery, in Medical Image Computing and Computer-Assisted Intervention (MICCAI'09), Lecture Notes in Computer Science (LNCS), London, UK, Springer, 2009, pp. 353-360.

[50] W. Wu and P.A. Heng, A hybrid condensed finite element model with gpu acceleration for interactive $3 \mathrm{~d}$ soft tissue cutting: Research articles, Comput. Animat. Virtual Worlds, 153-4 (2004), 219-227, ISSN 1546-4261.

[51] D. Zerbato, S. Galvan, and P. Fiorini, Calibration of mass spring models for organ simulations, in Intelligent Robots and Systems, 2007. IROS 2007. IEEE/RSJ International Conference on, 29 2007-Nov. 22007. 

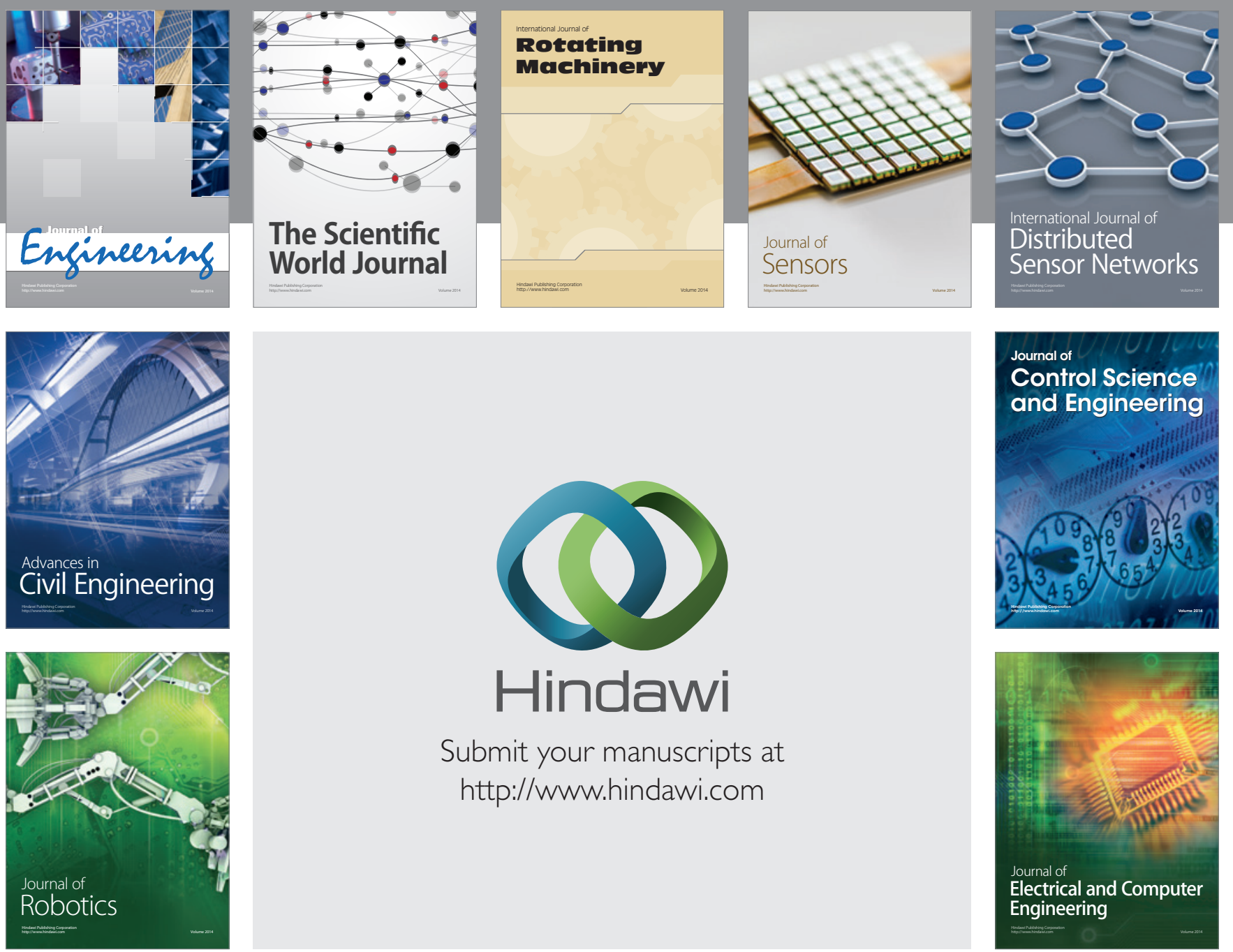

Submit your manuscripts at

http://www.hindawi.com
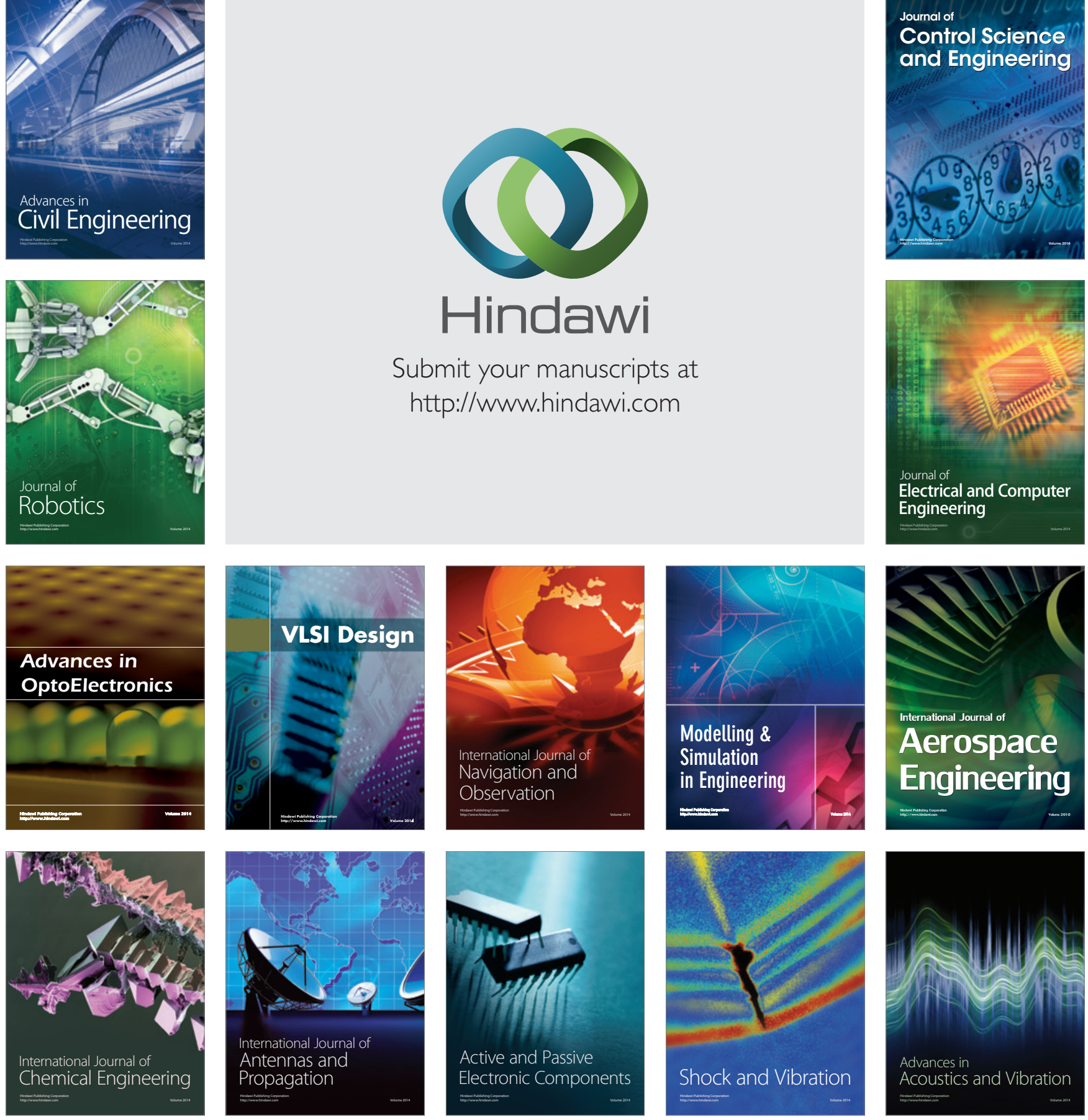